\title{
EVALUATION OF BIPHASIC CULTURE SYSTEM FOR MYCOBACTERIAL ISOLATION FROM THE SPUTUM OF PATIENTS WITH PULMONARY TUBERCULOSIS
}

\author{
*M Ghatole, C Sable, P Kamale, S Kandle, V Jahagirdar, V Yemul
}

\begin{abstract}
Mycobacterial diseases continue to cause high morbidity and mortality. Isolation, identification and sensitivity testing form the backbone of laboratory investigations. M. tuberculosis isolation needs 6-8 weeks on conventional egg containing media. For rapid isolation various methods have been evaluated. We evaluated biphasic system (Middlebrook 7H11 agar slant + Middlebrook 9H broth) in comparison with Lowenstein - Jensen (LJ) medium. In smear positive cases biphasic system showed the recovery rate of $97.05 \%$ as against $79.41 \%$ on LJ on incubation for $21 \pm 4.44$ and $28 \pm 3.76$ days respectively. In smear negative and culture positive cases biphasic system and $\mathrm{LJ}$ showed isolation rates of $91.66 \%$ and $66.6 \%$ after $36 \pm 3.44$ and $41 \pm 4.09$ days respectively. Biphasic system showed lower contamination rate (1.33\%). Biphasic medium is superior to $\mathrm{LJ}$ medium in isolation of M. tuberculosis.
\end{abstract}

Key words: M. tuberculosis, biphasic system, culture

Mycobacterial infections continue to cause high mortality. During the year 2000 about 1.66 million people died of tuberculosis and the global burden in terms of DALYs loss was about 35.79 million. India accounts for nearly one third of the global burden i.e., approximately 2.2 million cases. The incidence per lakh population is 184 with $3.4 \%$ multidrug resistant cases. ${ }^{1,2}$

In all mycobacterial infections, isolation of the aetiological agent forms the definitive investigation. Isolation of mycobacteria is also essential for drug sensitivity testing, epidemiological surveys, case finding and assessment of tuberculosis control programmes. For the isolation of $M$. tuberculosis conventional egg based media require up to 810 weeks of incubation. Thus there is a great need for developing rapid, sensitive, simple and economic culture methods. Introduction of radiometric system with use of selective broth medium in BACTEC 460 has improved the recovery rate and also decreased the incubation period but this system has limitations of being expensive along with disposal of radioactive waste. ${ }^{3}$ Similarly, other techniques like fluorescent BACTEC 9000, colorimetric system, MGIT system etc. were introduced. ${ }^{4-6}$ But in developing country like India, where disease burden is high, these sensitive techniques are not easily available.

The commercial biphasic system (septi chek AFB system or MB chek system) was introduced and evaluated for

*Corresponding author (email: <mangalghatole@hotmail.com>) Department of Microbiology, Dr. V. M. Medical College, Solapur 413 002, Maharashtra, India

Received: 06-01-2004

Accepted: 31-07-2004 improving the isolation rate and reducing the recovery time. ${ }^{7}$ In the present study we have evaluated the use of biphasic medium in our laboratory using Middlebrook 7H11 agar with OADC supplement (M511 FD018- Hi-Media) slant as solid phase and Middlebrook 9H broth with ADC supplement (M198, FD019-Hi-Media) as liquid phase i.e., MB biphasic system in comparison with conventional Lowenstein - Jensen (LJ) medium.

\section{Materials and Methods}

Between January and December 2002, a total of 151 patients having clinical and radiological evidence of tuberculosis attending the department of tuberculosis and medicine at SCSM hospital, Solapur, who had not taken antitubercular therapy, were selected.

From each patient sputum sample was collected in sterile wide mouthed container for three consecutive mornings and transported to the laboratory. The sputum samples were concentrated and decontaminated by Petroff's method. ${ }^{9}$

LJ medium was prepared by using LJ base obtained from Hi-Media (M162). All ingredients were dissolved by heating and the medium was autoclaved. This medium was mixed with $1000 \mathrm{~mL}$ of whole egg emulsion prepared aseptically and $20 \mathrm{~mL}$ of $1 \%$ malachite green solution. The complete medium was dispensed in McCartney bottles and inspissated at $80^{\circ} \mathrm{C}$ for one hour.

Middlebrook biphasic medium (MB system) was prepared in two stages. For solid phase, Middlebrook 7H11 agar base obtained from Hi-Media (M511) was used. Slants were prepared as per the manufactured instructions. For liquid phase 2.35 grams of dehydrated Middlebrook $7 \mathrm{H} 9$ broth base 
(Hi-Media, M 198) was dissolved in $450 \mathrm{~mL}$ distilled water containing $2 \mathrm{~mL}$ of glycerol by heating and autoclaved at $121^{\circ} \mathrm{C}$ for 15 minutes. Medium was cooled to $50^{\circ} \mathrm{C}$ and one vial of ADC supplement (Hi-Media, FD019) was added. Ten millilitre of this medium was poured over the formed slant.

Before proceeding with the culture of the clinical samples on both the media we did an initial quality control study by inoculating H 37Rv strain of M. tuberculosis, five strains of $M$. tuberculosis and two strains of $M$. kansasii isolated and identified in our laboratory.

From each concentrated and decontaminated sample 0.5 $\mathrm{mL}$ was inoculated on $\mathrm{LJ}$ medium, $0.5 \mathrm{~mL}$ on Middlebrook slant and $0.5 \mathrm{~mL}$ on Middlebrook broth. Biphasic medium was considered as two systems incorporated into one container so inoculations were done into both. Two smears were prepared on clean grease free slides and stained with Zeihl-Neelsen (ZN) stain and examined. The inoculated media were incubated aerobically at $37^{\circ} \mathrm{C}$. All bottles were inspected daily for the detection of growth. The biphasic medium was tilted (for inoculation of slant) on alternate days in the first two weeks, there after once a week.

If a growth was detected, its description along with the duration required was noted. Smears were prepared from the growth, stained with ZN stain and examined. The isolates were identified by conventional biochemical tests. ${ }^{10}$ If no growth was detected after eight weeks of incubation then smears were prepared from MB broth, MB slant, LJ slant and

Table 1: Showing detection of M. tuberculosis with different methods

\begin{tabular}{|c|c|c|}
\hline Methods & Total positivity & Recovery rate* \\
\hline Direct smear & $34(22.51 \%)$ & $73.09 \%$ \\
\hline LJ medium & $35(23.17 \%)$ & $76.08 \%$ \\
\hline Biphasic medium & $44(29.13 \%)$ & $95.65 \%$ \\
\hline
\end{tabular}

* Recovery rate is determined by the proportion of total number of mycobacteria detected by each method. examined before reporting as culture negative.

Both the culture methods were compared regarding their recovery rate, duration needed and contamination rate.

\section{Results}

Among the 151 sputum samples 46 (30.46\%) detected the organism M. tuberculosis by one or both methods. Thirty four $(23.5 \%)$ of the samples showed presence of acid fast bacteria in the smear. Thirty four cultures were isolated by both methods. One culture was grown only on LJ and 10 only on biphasic system. Sensitivity and specificity of biphasic system was $97.43 \%$ and $93.27 \%$ respectively. Table 1 summarizes the rate of recovery of $M$. tuberculosis by each method. MB biphasic system showed recovery rate of $97.05 \%$ and LJ $79.41 \%$ in smear positive cases. In smear negative cases MB biphasic system yielded $91.66 \%$ and LJ $66.66 \%$. According to McNemar's modification of $\mathrm{X}^{2}$ test the biphasic medium gave significant $(P<0.05, P<0.001)$ results in smear positive and negative cases respectively. The over all mean time required for recovery of $M$. tuberculosis was $21 \pm 4.44$ days and $28 \pm 3.76$ days in smear positive cases, $36 \pm 3.44$ days and $41 \pm 4.09$ days in smear negative cases by biphasic system and LJ medium respectively (table 1 and 2).

\section{Discussion}

Resurgence of tuberculosis due to HIV pandemic and multidrug resistant mycobacterial strains has renewed interest in developing more efficient system for isolation, identification and drug susceptibility testing of $M$. tuberculosis.

In our study of 151 sputum samples the over all detection was $46(30.46 \%)$ by one or more methods. The recovery rate of M. tuberculosis on biphasic medium was $44(95.65 \%)$ and $35(76.08 \%)$ on LJ. Similar observations were made by Luquin et $a l^{11}$ who has shown recovery rate of $92.5 \%$ and $79.6 \%$ by MB chek and LJ medium respectively. In their study they have also shown MB chek to be superior to BACTEC. In contrast, Piersimoni et $a l^{12}$ have shown BACTEC to be superior $(91.9 \%)$ to $\mathrm{MB}$ chek $(79.7 \%)$. Abe et $a l^{13}$ have

Table 2: Comparison of LJ medium and biphasic method

\begin{tabular}{lcccccc}
\hline & \multicolumn{3}{c}{ Biphasic medium } & & \multicolumn{2}{c}{ LJ medium } \\
\cline { 2 - 3 } & Recovery rate & $\begin{array}{c}\text { Duration } \\
\text { (days) }\end{array}$ & Contamination & Recovery rate & $\begin{array}{c}\text { Duration } \\
\text { (days) }\end{array}$ & Contamination \\
\hline $\begin{array}{l}\text { Smear and } \\
\text { culture positive }\end{array}$ & $33(97.05 \%)$ & $21 \pm 4.44$ & $2(1.32 \%)$ & $27(79.41 \%)$ & $28 \pm 3.76$ & $7(4.63 \%)$. \\
$\begin{array}{l}\text { Smear } \\
\text { negative } \\
\text { and culture positive }\end{array}$ & $11(91.66 \%)$ & $36 \pm 3.44$ & $8(66.66 \%)$ & $41 \pm 4.09$ \\
\hline
\end{tabular}


reported same recovery rates of $M$. tuberculosis by both septi - chek and BACTEC.

We evaluated recovery rates of $M$. tuberculosis among smear positive and smear negative samples (table 2). Our observations are similar to Sewell et $a l^{14}$ and Luquin et al. ${ }^{11}$ We emphasize the difference in recovery rates of two methods in smear negative cases. Similar observations were also made by Ichiyama et al..$^{15}$

The average number of days required for the recovery of M. Tuberculosis by both culture methods in smear positive and smear negative cases observed by us are similar to those reported by Sewell et al ${ }^{14}$ but Luquin et al ${ }^{11}$ observed shorter incubation period.

Evaluation of any method is incomplete without taking into consideration the contamination rate. We observed $4.63 \%$ contamination on LJ medium and only $1.3 \%$ on biphasic system, which is consistent with that reported by Luquin et $a l .{ }^{11}$ However, Isenberg et $a l^{16}$ have shown higher contamination rate with septi-chek $(4.5 \%)$ than $\mathrm{LJ}$ medium $(2 \%)$.

Cost of MB biphasic system was calculated to be 1.8 times the cost of LJ medium when prepared indigenously in our laboratory. We did not come across any commercial company in India preparing biphasic system for M. tuberculosis. So we could not compare the cost of commercially available media.

In conclusion, we observed the performance of biphasic system to be superior in recovering $M$. tuberculosis. Ease of processing the sample and not requiring the special expensive equipment or radioactive media, are its advantages. The biphasic system is feasible and practical method even in medium sized laboratories. It has the advantage of repeated exposure of agar medium to actively proliferating organisms in the liquid broth during subculturing, which is simply by tilting the bottle. In this system different combinations of media can be used for better differentiation of mycobacteria.

\section{References}

1. Govt of India DGHS Annual report 2001-2002. New Delhi.

2. WHO Weekly Epidemiological record 2002.

3. Bhargava A, Jain A, Agarwal SK. A comparison of liquid and solid culture media with radiometric system for detection of mycobacteria in clinical specimens. Indian J Tub 2001;48:9-12.

4. Zanetti S, Ardito T, Sechi M, Sanguineth P. Evaluation of non radiometric system (BACTEC $9000 \mathrm{MB}$ ) for detection Mycobacteria in human clinical samples. J Clin Microbiol 1997;35:2072-5.

5. Mahadev B, Srikantaramu N, James P, Mathew PG, Bhagirathi $\mathrm{R}$. Comparison between rapid colorimetric mycobacterial isolation and susceptibility testing method and conventional method using LJ medium. Indian J Tub 2001;48:129-34.

6. Chitra C, Prasad CE. Evaluation of Mycobacterial growth indicator tube (MGIT) for primary isolation of mycobacteria from clinical specimens. Indian J Tub 2001;48:155-6.

7. D' Amato RF, Isenberg HD, Houchstein L. Evaluation of the Roche Septi-chek AFB system for recovery of mycobacteria. $J$ Clin Microbiol 1991;29:2906-8.

8. Lwanga SK, Lemeshao S. Sample size determination in health studies - A Practical manual WHO publications 1991. p. 25.

9. Colle JG, Duguid JP, Fraser AG, Marimon BP. Mackie and MacCartney. 13 ${ }^{\text {th }}$ Ed. Practical Med Microbiol 1985;2:399.

10. Collins CH, Grange JM, Yates MD. Organization and Practice in Tuberculosis Bacteriology 1985. p. 60-5.

11. Luquin M, Gamboa F, Barcelo MG, Manterola JM, Matas L, Gimenez M, et al. Comparison of a biphasic non radiometric system with Lowenstein Jensen and BACTEC 460 system for recovery of mycobacteria from clinical samples. Tuber Lung Dis 1996;77:449-53.

12. Pierisonini C, Morbiducci V, De Sio G, Scalise G. Comparative evaluation of the MB chek system for recovery of mycobacteria from clinical specimens. Eur J Clin Microbiol Infect Dis 1992;11:1174-7.

13. Abe C, Hosojima S, Fukasawa Y, Kazumi Y, Takahashi S, Hirano K, et al. Comparison of MB chek, BACTEC and egg based media for recovery of mycobacteria. J Clin Microbiol 1992;30:878-81.

14. Sewell DL, Rashad AL, Rourke WJ, Sharon Jr, Poor L, McCarthy JA, et al. Comparison of Septi-chek AFB and BACTEC system and conventional cultures for recovery of mycobacteria. J Clin Microbiol 1993;31:2689-91.

15. Ichiyama S, Shimokota K, Takenchi J, et al. Comparative study of a biphasic culture system (Roche MB chek system) with a conventional egg medium for recovery of mycobacteria. Tuber Lung Dis 1993;74:338-41.

16. Isenberg HD, D'Amito RF, Heifets L, Murray PR, Scardamaglia $\mathrm{V}$, Jacob M, et al. Collaborative feasibility study of a biphasic system (Roche Septi chek AFB) for rapid detection and isolation of mycobacteria. J Clin Microbiol 1991;29:1719-922. 